\title{
Positive Correlation of Fitness with Group Size in Two Biotypes of Russian Wheat Aphid (Homoptera: Aphididae)
}

\author{
J. P. MICHAUD, ${ }^{1}$ J. L. JYOTI, AND J. A. QURESHI \\ Department of Entomology; Kansas State University; Agricultural Research Center-Hays; 1232 240th Avenue,
} Hays, KS 67601

\begin{abstract}
J. Econ. Entomol. 99(4): 1214-1224 (2006)
ABSTRACT Changes in fitness parameters as a function of colony size (one versus 10 aphids) were measured in two biotypes (RWA1 and RWA2) of the Russian wheat aphid, Diuraphis noxia (Mordvilko) (Homoptera: Aphididae), feeding on three cultivars of wheat, Triticum aestivum L., at two temperatures. 'Trego' is a cultivar with no specific resistance to D. noxia, whereas, 'Stanton' and 'Halt' express Dny and Dn4 resistance sources, respectively. Feeding in a group accelerated the development of RWA1 on Trego and Stanton at $20^{\circ} \mathrm{C}$, but not at $24^{\circ} \mathrm{C}$, whereas grouped RWA2 developed faster than solitary RWA2 on all three cultivars at $24^{\circ} \mathrm{C}$, but not at $20^{\circ} \mathrm{C}$. Survival (first instar-adult) of RWA2 also was improved by grouping on Stanton and Halt at $24^{\circ} \mathrm{C}$, but solitary RWA2 survived better at $20^{\circ} \mathrm{C}$ on all three cultivars. The reproductive rate of RWA1 was improved by grouping on Trego and Stanton at both temperatures, but only on Halt at $24^{\circ} \mathrm{C}$. Lifetime fecundity of RWAl also was increased by grouping in all cases except for Trego at $20^{\circ} \mathrm{C}$. Grouped development increased the reproductive rate of RWA2 on all three cultivars at $24^{\circ} \mathrm{C}$, but had no effect at $20^{\circ} \mathrm{C}$. Grouped RWA2 developed and reproduced faster than grouped RWAl on all three cultivars at $24^{\circ} \mathrm{C}$. Thus, the fitness of $D$. noxia was positively correlated with group size during colony establishment, but the effects were sensitive to temperature, being more pronounced at $20^{\circ} \mathrm{C}$ for RWA1 and at $24^{\circ} \mathrm{C}$ for RWA2.
\end{abstract}

KEY WORDS development, Diuraphis noxia, reproduction, survival, temperature

Aphids feed exclusively from plant phloem elements, processing large amounts of plant sap to obtain sufficient nitrogen for growth and reproduction and excreting excess carbohydrates in the form of honeydew (Dixon 2000). Most aphids inject some form of saliva into the phloem of their host plant both before and during feeding. The primary function of aphid saliva is likely to inhibit normal sieve tube responses to damage and enable aphid feeding to proceed without the plant sealing off the phloem (Tjallingii 1995, Powell 2004). However, the saliva of some aphids also can trigger significant changes in host plant morphology that directly benefit the aphids (Miles 1998, 1999). Various aphids (e.g., Pemphigus spp.) induce the formation of galls that increase the surface area suitable for aphid feeding while simultaneously enclosing the colony and affording it physical protection (Stone and Schönrogge 2003). Intermediate, semigalling plant responses are induced by other aphids, e.g., the "rosetting" of foliage caused by Aphis spiraecola Patch feeding on Citrus sinensis L. (Michaud 2000) and the leaf rolling response induced by $D$. noxia on susceptible wheat (Randolph et al. 2003), both examples of aphid manipulation of host plant morphology that produce a

${ }^{1}$ Corresponding author, e-mail: jpmi@ksu.edu. more protected microenvironment for aphid colony growth and development.

The saliva of some aphids [e.g., Schizaphis graminum (Rondani), Myzus persicae (Sulzer), and Acyrthosiphum pisum (Harris)] has been shown to contain protein fractions with enzymatic activity (Cherqui and Tjallingii 2000), and these may often function to subvert host plant metabolism for the aphid's benefit (Dorschner et al. 1987, Sandström et al. 2000). For D. noxia, saliva injected into a susceptible host plant accelerates chlorophyll catabolism in photosynthetic tissues ( $\mathrm{Ni}$ et al. 2001), resulting in a concomitant increase in the concentration of free amino acids in phloem elements (Telang et al. 1999) and oxidative stress that ultimately results in plant death $(\mathrm{Ni}$ and Quisenberry 2003). Collectively, the aphids subvert normal metabolism to increase plant suitability for their own growth and reproduction. Because this involves the injection of a quantity of aphid saliva over some period, and a gradual response by the plant to elicitors within the saliva, it follows that the process should be more effective, and occur more rapidly, when aphids are able to pool their efforts in a group. Thus, we reasoned that induction of plant suitability by $D$. noxia should be dosage-dependent, i.e., multiple aphids should elicit changes in plant suitability more effectively than single aphids. If so, the improved nu- 
tritional value of multiply-induced plants should be reflected in superior aphid survival, development, and reproduction.

Bonnemaison (1951) first coined the term "effect of group" within aphid colonies when he observed that degree of crowding exerted a substantial influence on the development of alate morphs in both $M$. persicae and Brevicoryne brassicae (L.). Way (1967) showed that Aphis fabae Scopoli reproduced more quickly when in groups of eight than in either smaller or larger groups. This result was attributed to a "sink effect" caused by aphids feeding together that became negated by intraspecific competition in larger groups when the sink effect exceeded the plant's capacity to respond. Similarly, Way and Cammell (1970) found that $B$. brassicae developed into larger adults on a cabbage leaf when it had other aphids feeding on the opposite surface. At that time, such effects were inferred to result simply from increased flow rates of sap to the feeding site, rather than the induction of any qualitative changes in the phloem contents.

Cultivars of wheat vary in their suitability as food for D. noxia (Ali et al. 1985, Jyoti et al. 2006) and various "biotypes" of $D$. noxia are recognized based on their ability to damage cultivars expressing particular genetic sources of resistance (Basky 2003, Haley et al. 2004). For example, a series of commercial cultivars bred specifically for resistance to $D$. noxia express a gene designated Dn4, and these cultivars have been widely planted in regions of the United States where D. noxia has persistently caused economic losses (Randolph et al. 2003, CASS 2004), whereas other sources of resistance such as Dny have not been planted as widely. Although the actual mechanisms of resistance are not well understood, we hypothesized that cultivars expressing resistance to $D$. noxia might interfere with the ability of the aphids to elicit changes in host plant suitability and that such an effect would likely be reflected in any dosage-dependent effects of aphid feeding on their development and reproduction.

Previously, Jyoti and Michaud (2005) compared the performance of two $D$. noxia biotypes (now designated RWA1 and RWA2) on three wheat cultivars and noted significant differences. 'Trego' is a hard white winter wheat released by Kansas State University in 1999 that is highly susceptible to D. noxia (Jyoti et al. 2006). 'Halt' is a cultivar developed by Colorado State University and released in 1994 that was the first commercial cultivar expressing the Dn4 gene for D. noxia resistance (Liu et al. 2001). 'Stanton' was developed by Kansas State University and released in 2000 (Martin et al. 2001). This cultivar expresses a presumably different genetic source of $D$. noxia resistance, currently designated Dny (Smith et al. 2004).

RWA1 aphids performed better on the susceptible Trego (PI 612576) than on either of the two resistant cultivars, Stanton (PI 617033) and Halt (PI 584505), whereas RWA2 aphids performed well on all three cultivars and formed larger colonies even on Trego. In the current study, we designed two series of experiments to detect possible group feeding effects on the development and reproduction of both $D$. noxia bio- types on the same three cultivars, with temperature as an additional independent variable. We hypothesized that, if aphids feeding together on a plant are able to collectively enhance plant suitability better than single aphids, then groups of first instars should survive better, and develop to maturity faster, than solitary first instars. Furthermore, we predicted that adult apterae developing in groups and then reproducing on the same multiply-induced plant would achieve greater reproductive success than those developing and reproducing on singly-induced plants. We also hypothesized that these group effects would be substantially reduced for RWA1 on the cultivars expressing resistance to this biotype.

\section{Materials and Methods}

Insect Colonies. A colony of D. noxia, RWA1 was established from aphids collected in Hays, KS, in 2002 and maintained in continuous culture on T. aestivum Trego in a climate-controlled growth chamber at $22^{\circ} \mathrm{C}$. Lighting was provided with banks of Philips coolwhite fluorescent set to a photoperiod of 16:8 (L:D) h. The identity of this colony as RWAl was confirmed in repeated experiments that demonstrated inferior performance on the Dn4-expressing cultivars Halt, 'Prairie Red', and 'Yumar' (Jyoti and Michaud 2005, Jyoti et al. 2006). A colony of $D$. noxia, RWA2 was established from material obtained from USDA-ARS, Stillwater, $\mathrm{OK}$, originally collected in eastern Colorado in spring 2003, and maintained on T. aestivum Halt under the same environmental conditions as RWA1. The identity of this colony as RWA2 was confirmed in repeated experiments that demonstrated its performance on Dn4-expressing cultivars was not reduced compared with its performance on the susceptible Trego.

We used Trego for maintaining RWA1, because it is a highly suitable cultivar for rearing this biotype and expresses no specific resistance to RWA. Halt was used for maintaining RWA2, because it expresses Dn4 resistance and therefore selects for continued virulence to Dn4 in the RWA2 colony. Colonies of the two biotypes were held in different buildings, and careful quarantine procedures were observed to prevent cross-contamination, including the fumigation of all greenhouse-grown wheat plants with dichlorvos before infestation with aphids (fumigated plants were aired for $24 \mathrm{~h}$ before infestation), and the use of biotype-specific laboratory coats in each building whenever aphids were handled.

Experimental plants of each cultivar (Trego, Stanton, and Halt) were seeded individually in plastic cones (Stuewe \& Sons, Corvallis, OR) and germinated in a greenhouse. Plants were infested at the two-leaf stage (9-10 d old, Zadoks growth stage 12), and each was then covered with a ventilated, clear plastic cylinder creating a "cone-tainer" to isolate the developing aphid(s) following the technique pioneered by Harvey and Kofoid (1993) and modified by Jyoti and Michaud (2005). 
Development Assay. First instar aphids ( $\pm 12 \mathrm{~h}$ old) of each biotype were obtained by placing reproductive apterous adult aphids on wheat seedlings and permitting them to reproduce for a period of $24 \mathrm{~h}$. The first instars were then transferred to experimental plants individually using a moistened camel hair brush. The "solitary" treatment consisted of one first instar on each experimental plant and the "grouped" treatment consisted of 10 first instars on each plant. Because we anticipated differential survival of solitary aphids according to wheat cultivar (Jyoti and Michaud 2005), we infested different numbers of plants according to biotype and cultivar so that roughly similar sample sizes of single aphids would complete development on all three cultivars. For RWA1, a total of 25 replicates of the solitary treatment were initiated on Trego, and 40 replicates on both Stanton and Halt. For RWA2, we had 40 replicates on Trego and 25 each on Stanton and Halt. For the grouped treatment, 20 replicates were initiated on each cultivar for both biotypes. The entire experiment was repeated for each biotype at constant temperatures of 20 and $24^{\circ} \mathrm{C}$. This yielded an overall experimental design with four independent variables: biotype (two) $\times$ grouping treatment (two) $\times$ cultivar (three) $\times$ temperature (two). Beginning $5 \mathrm{~d}$ after infestation, each experimental replicate was examined daily to determine the number of aphids alive. Each adult aphid was removed the day it matured and began depositing nymphs, and the developmental time was calculated as the number of days from birth to deposition of the first nymph. This procedure enabled us to compare the developmental time of aphids that matured on a plant alone versus in groups of 10 aphids. Data for each biotype-cultivar combination at each temperature were first analyzed pairwise for effects of the grouping treatment and then by fully factorial analysis of variance (ANOVA) to detect interactions between temperature, biotype, cultivar, and grouping treatment (SAS Institute 1999-2000). Means were separated by multiple $t$-tests obtained from the least square means statement of GLM.

Reproduction Assay. Preparation of plants and aphids was identical to that of the development assay, and the same experimental array of cultivars and treatments was used and replicated at both 20 and $24^{\circ} \mathrm{C}$. Experimental procedures were also identical up to the point of aphid maturity. Maturity was again tallied as the day an aphid produced its first nymph, but in the grouped treatment the first aphid to deposit a nymph was selected for reproductive observations, and all other aphids were tallied and removed from the plant. All nymphs deposited by reproducing aphids in both the solitary and grouped treatments were tallied and removed daily until the death of the adult. Lifetime fecundity was determined for each aphid as the total number of nymphs produced. Reproductive life span was calculated as the number of days from deposition of the first nymph to deposition of the last nymph.

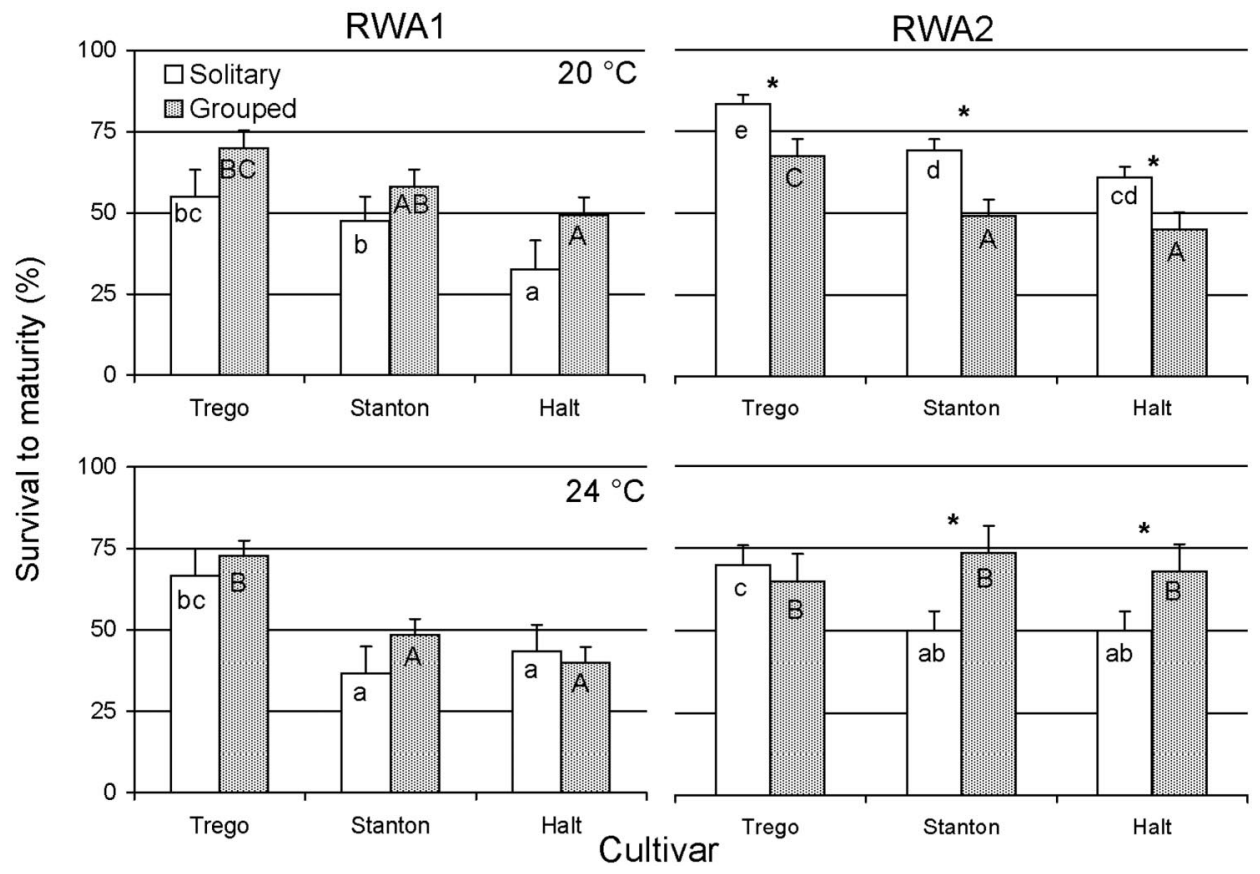

Fig. 1. Mean survival rates to adulthood of two D. noxia biotypes (RWA1 and RWA2) reared on three wheat cultivars at two constant temperatures. Solitary individuals developed in isolation on a wheat seedling; grouped individuals developed in groups of 10. Asterisks indicate significant differences (ANOVA, $\alpha<0.05$ ) between solitary and grouped aphids in a particular biotype-cultivar-temperature combination. Different lowercase letters indicate values for solitary aphids that were significantly different $(\alpha<0.05)$ among biotype-cultivar combinations within a temperature treatment; different uppercase letters indicate values for grouped aphids that were significantly different among biotype-cultivar combinations with in a temperature treatment. 


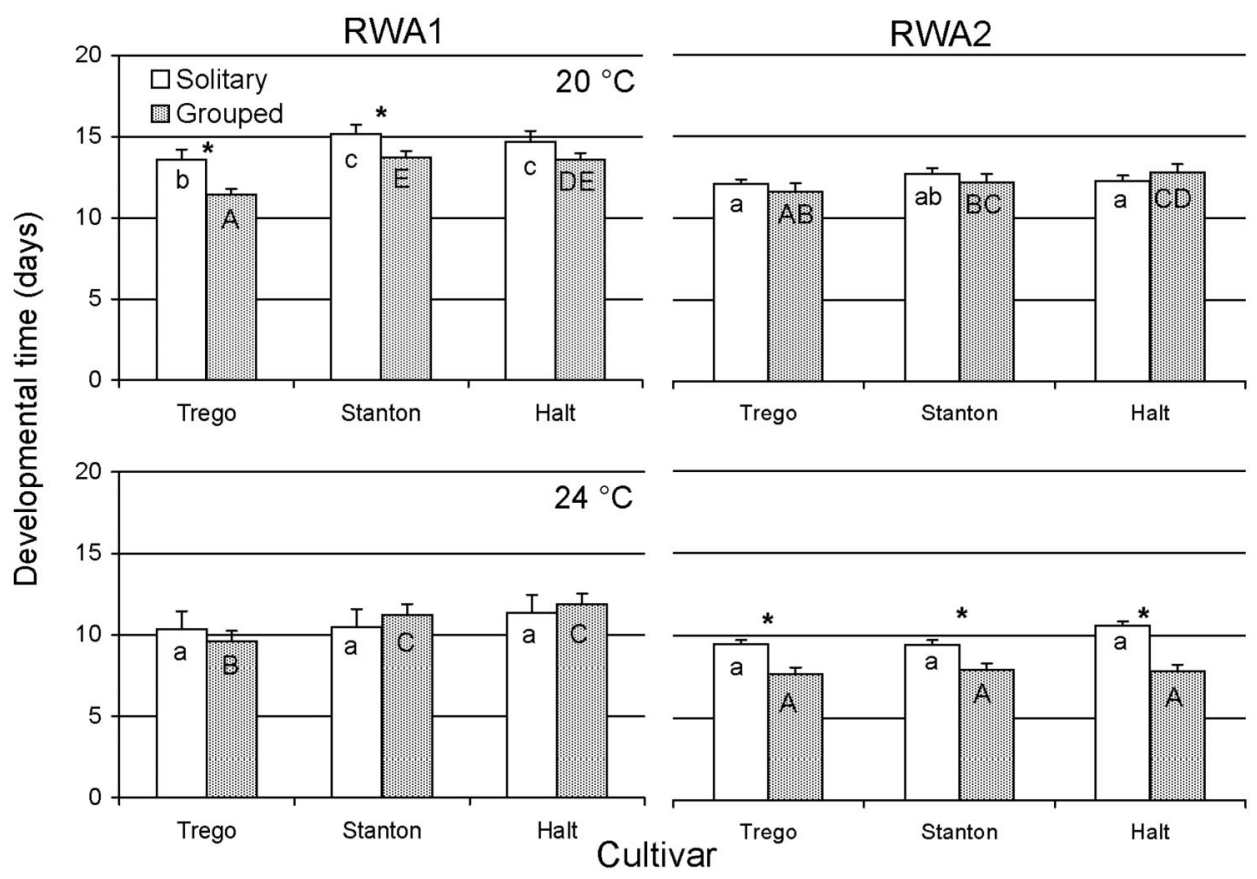

Fig. 2. Mean developmental times (+SEM) of two D. noxia biotypes (RWA1 and RWA2) reared on three wheat cultivars at two constant temperatures. Solitary individuals developed in isolation on a wheat seedling; grouped individuals developed in groups of 10. Asterisks indicate significant differences (ANOVA, $\alpha<0.05$ ) between solitary and grouped aphids in a particular biotype-cultivar-temperature combination. Different lowercase letters indicate values for solitary aphids that were significantly different $(\alpha<0.05)$ among biotype-cultivar combinations within a temperature treatment; different uppercase letters indicate values for grouped aphids that were significantly different among biotype-cultivar combinations within a temperature treatment.

Reproductive rate was calculated for each aphid by dividing its lifetime fecundity by its reproductive life span. These data were then analyzed as for the development assay. In addition, we used linear regression to establish relationships between independent variables, and between group size and developmental time, fecundity, and reproductive rate within the grouped treatment.

\section{Results}

Development Assay. Actual temperatures recorded in growth chambers over the course of the experiment averaged 19.95 and $20.78^{\circ} \mathrm{C}$ for RWA1 and RWA2, respectively, in the $20^{\circ} \mathrm{C}$ treatment and 24.05 and $24.54^{\circ} \mathrm{C}$, respectively, in the $24^{\circ} \mathrm{C}$ treatment. Survival rates for solitary and grouped aphids are compared among all six biotype-cultivar combinations at each temperature in Fig. 1 and their developmental times, in Fig. 2. There were significant effects of biotype and cultivar on aphid survival to maturity, and significant effects of biotype, temperature, cultivar, and grouping on the developmental times of surviving aphids (Table 1 ). The lack of a significant four-way interaction (biotype $\times$ cultivar $\times$ temperature $\times$ grouping) is an indication that the relative performance of each biotype within a grouping treatment was independent with respect to its developmental time and survival irrespective of temperature or cultivar. The only sig- nificant three-way interaction for developmental time and survival (biotype $\times$ grouping $\times$ temperature) indicated that these variables did not respond independently across cultivars. There were significant two-way interactions between biotype and cultivar for developmental time and between biotype and grouping and grouping and temperature for aphid survival.

Reproduction Assay. Actual temperatures recorded in growth chambers over the course of the experiment averaged 20.09 and $20.02^{\circ} \mathrm{C}$ for RWA1 and RWA2, respectively, in the $20^{\circ} \mathrm{C}$ treatment and 24.42 and $24.13^{\circ} \mathrm{C}$, respectively, in the $24^{\circ} \mathrm{C}$ treatment. Mean values for fecundity, reproductive life span, and reproductive rate are compared for solitary and grouped aphids among all six biotype-cultivar combinations at each temperature in Figs. 3 and 4, and 5, respectively. There were significant effects of biotype, cultivar and grouping on both fecundity and reproductive rate, and significant effects of biotype on reproductive life (Table 2). There was a significant four-way interaction (biotype $\times$ grouping $\times$ temperature $\times$ cultivar) for fecundity, indicating that the number of offspring produced by females was influenced by interactions between all independent variables. The same was not true for reproductive rate or reproductive life. However, there were significant two-way interactions between biotype and cultivar for fecundity, reproductive life, and reproductive rate, indicating that these dependent variables did not respond independently 
Table 1. ANOVA of developmental time and proportional survival to adult for $D$. noxia reared from first instar either in solitude or in groups of 10 (=grouping treatment) on each of three wheat cultivars (Trego, Stanton, and Halt) at each of two temperatures (20 and $\left.24^{\circ} \mathrm{C}\right)$

\begin{tabular}{|c|c|c|c|c|c|}
\hline \multirow{2}{*}{ Source of variation } & \multirow{2}{*}{$\mathrm{df}$} & \multicolumn{2}{|c|}{ Developmental time (d) } & \multicolumn{2}{|c|}{ Survival } \\
\hline & & $F$ & $P$ & $F$ & $P$ \\
\hline Biotype & 1 & 23.58 & 0.0083 & 87.60 & 0.0007 \\
\hline Replication & 2 & 0.77 & 0.5968 & 10.14 & 0.0227 \\
\hline Replication (clone) $=$ error $(\mathrm{a})$ & 4 & 5.41 & 0.0004 & 0.27 & 0.8993 \\
\hline Temperature & 1 & 260.17 & 0.0001 & 0.09 & 0.7760 \\
\hline Biotype*temp & 1 & 2.05 & 0.1905 & 0.01 & 0.9243 \\
\hline Rep*temp $($ clone $)=$ error $(b)$ & 8 & 1.70 & 0.0998 & 0.70 & 0.6910 \\
\hline Cultivar & 2 & 12.27 & 0.0001 & 25.02 & 0.0001 \\
\hline Grouping treatment & 1 & 19.08 & 0.0001 & 2.47 & 0.1198 \\
\hline Cultivar*grouping treatment & 2 & 1.18 & 0.3113 & 0.32 & 0.7305 \\
\hline Biotype*cultivar & 2 & 2.42 & 0.0956 & 0.88 & 0.4188 \\
\hline Biotype*grouping treatment & 1 & 0.93 & 0.3367 & 4.81 & 0.0312 \\
\hline Temp*cultivar & 2 & 1.63 & 0.2022 & 0.70 & 0.4987 \\
\hline Temp* grouping treatment & 1 & 0.02 & 0.8921 & 3.50 & 0.0649 \\
\hline Biotype*cultivar*grouping treatment & 2 & 0.63 & 0.5337 & 0.52 & 0.5951 \\
\hline Biotype*temp*cultivar & 2 & 0.23 & 0.7965 & 3.28 & 0.0428 \\
\hline Biotype*temp*grouping treatment & 1 & 19.94 & 0.0001 & 13.93 & 0.0004 \\
\hline Temp*cultivar*grouping treatment & 2 & 0.97 & 0.3822 & 1.93 & 0.1526 \\
\hline Biotype*temp*cultivar*grouping treatment & 2 & 0.62 & 0.5414 & 1.27 & 0.2861 \\
\hline Rep*trt (biotype*temp*cultivar) $=$ error $(\mathrm{c})$ & 80 & 1.84 & 0.0003 & 1.12 & 0.2575 \\
\hline
\end{tabular}

Rep, replicate; trt, treatment.

across temperature and grouping treatment. The interaction between cultivar and grouping was significant for reproductive rate, but not for reproductive life or fecundity, whereas the interaction between biotype and cultivar was significant for all three dependent variables.

Fecundity was positively correlated with reproductive life span at both $20^{\circ} \mathrm{C}$ (RWAl: $F=128.80, P<$ $0.001, r^{2}=0.490$; RWA2: $F=245.19, P<0.001, r^{2}=$ 0.610 ) and $24^{\circ} \mathrm{C}$ (RWA1: $F=288.12, P<0.001, r^{2}=$ 0.634; RWA2: $F=581.52, P<0.001, r^{2}=0.780$ ). Reproductive rate was negatively correlated with reproductive life span at $20^{\circ} \mathrm{C}$ (RWAl: $F=5.04, P=$ 0.026, $r^{2}=0.029$; RWA2: $F=10.1, P=0.002, r^{2}=$ 0.058 ), but not at $24^{\circ} \mathrm{C}$ (RWA1: $F=0.11, P=0.738$; RWA2: $F=1.97, P=0.163$.

The size of the aphid group at the time of first reproduction was positively correlated with the fecundity and reproductive rate of the first-maturing RWAl at both temperatures and negatively correlated with its developmental time (Table 3 ). In contrast, no regressions were significant for RWA2 at either temperature.

\section{Discussion}

The two aphid colonies we examined made up parthenogenetically propagated clones originally established from single virginoparae and clearly represented two distinct biotypes of D. noxia. However, it should be noted that variation is likely to exist among clones within biotypes (Shufran et al. 1992), because biotypic designations are made solely on the basis of plant interactions. Furthermore, various clones of RWA2 have been observed to vary in their ability to damage wheat plants (K. A. Shufran, personal communication). It must be stressed that all biotype-spe- cific results reported here apply specifically to these $D$. noxia clones and are not necessarily representative of the full range of responses that might be observed in natural populations, although we expect the generalized effects of the grouping treatment would hold true for all clones.

The reduced performance of RWAl observed on both resistant cultivars is consistent with previous work (Haley et al. 2004, Jyoti and Michaud 2005, Jyoti et al. 2006). The juvenile survival of RWAl was reduced on both resistant cultivars at $24^{\circ} \mathrm{C}$, although only on Halt at $20^{\circ} \mathrm{C}$, with the same pattern evident for both singles and groups. Developmental time of RWA1 was lengthened for both singles and groups on resistant cultivars at $20^{\circ} \mathrm{C}$, but only for groups at $24^{\circ} \mathrm{C}$. However, these resistance sources had their greatest impact on aphid reproduction, reducing fecundity at $20^{\circ} \mathrm{C}$ by an average of 63 and $46 \%$ for solitary and grouped RWA1, respectively, and at $24^{\circ} \mathrm{C}$ by 64 and $50 \%$, respectively. Reproductive rate was reduced on the resistant cultivars by an average of 32 and $41 \%$ at $20^{\circ} \mathrm{C}$ for solitary and grouped RWA1, respectively, and by 44 and $47 \%$, respectively, at $24^{\circ} \mathrm{C}$. In addition, the reproductive lifespan of solitary RWA1 was shortened on the resistant cultivars at both temperatures, although this was not the case for their counterparts in groups. These cultivar effects seemed relatively consistent between temperatures and grouping treatments, suggesting that neither temperature nor grouping of aphids had much effect on the expression of resistance to RWA1.

Certain reductions in the performance of RWA2 were also evident on the resistant cultivars, but mostly at $20^{\circ} \mathrm{C}$, the temperature that seemed to impede induction of plant suitability by this biotype. Both solitary and grouped RWA2 had reduced juvenile survival on the resistant cultivars at $20^{\circ} \mathrm{C}$, but this was only 


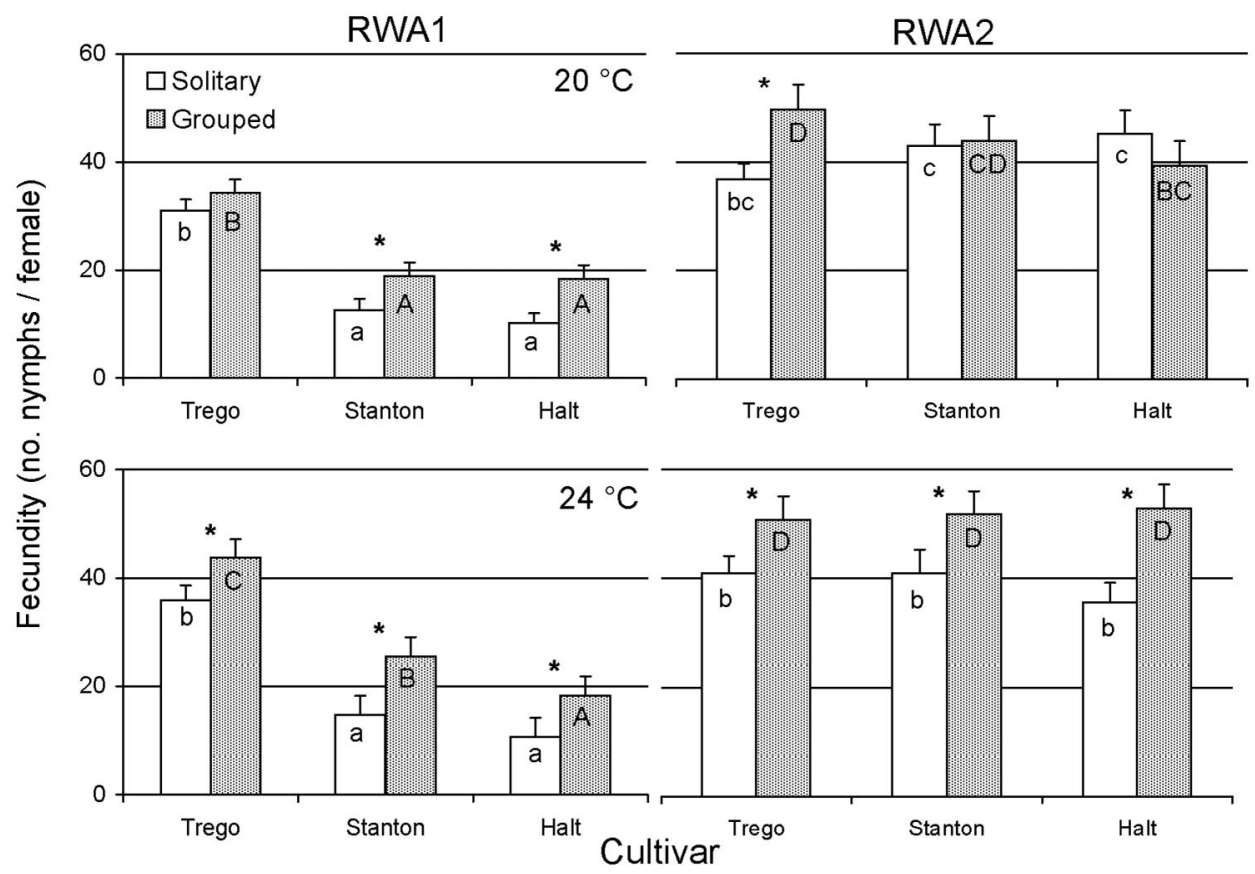

Fig. 3. Mean fecundities (+SEM) of two D. noxia biotypes (RWA1 and RWA2) reared on three wheat cultivars at two constant temperatures. Solitary individuals developed in isolation on a wheat seedling and reproduced alone; grouped individuals developed in groups of 10 , and the first to mature was left on the plant to reproduce alone. Asterisks indicate significant differences (ANOVA, $\alpha<0.05$ ) between solitary and grouped aphids in a particular biotype-cultivar-temperature combination. Different lowercase letters indicate values for solitary aphids that were significantly different $(\alpha<0.05)$ within a temperature treatment; different uppercase letters indicate values for grouped aphids that were significantly different within a temperature treatment.

true for solitary RWA2 at $24^{\circ} \mathrm{C}$. Development was slightly faster for grouped RWA2 on Trego than on Halt at $20^{\circ} \mathrm{C}$, but other differences in developmental time were not significant among cultivars at either temperature when solitary and grouped aphids were considered separately. Grouped RWA2 had lower fecundity and reproductive rate on Halt than on Trego at $20^{\circ} \mathrm{C}$, but there were no differences among cultivars in fecundity at $24^{\circ} \mathrm{C}$, although the reproductive rate of solitary RWA2 was higher on Trego compared with Stanton or Halt at this temperature. The reproductive life span of solitary RWA2 was greater on Halt than on Trego at $20^{\circ} \mathrm{C}$, but other differences in reproductive life span were not significant at either temperature, regardless of whether singles or groups were compared. Thus, RWA2 was more generally effective at negating the resistance of Stanton and Halt at the higher temperature.

Grouped RWA2 had consistently faster development than grouped RWAl on all cultivars at $24^{\circ} \mathrm{C}$, whereas the same was not true for solitary aphids. RWA2 tended to have longer reproductive life spans than RWAl on all cultivars at $20^{\circ} \mathrm{C}$, regardless of whether solitary or grouped aphids were compared, a factor that enabled it to achieve significantly higher fecundities than RWA1 at this temperature despite similar rates of reproduction. RWA2 also achieved higher fecundities than RWAl on all cultivars at $24^{\circ} \mathrm{C}$, but in this case it was the result of higher rates of reproduction without any increases in reproductive life span. Again, these results are consistent with RWA2 more effectively overcoming resistance at the higher temperature.

Responses of the two D. noxia biotypes to the grouping treatment diverged across the two temperature regimes in both experiments. Although the beneficial effects of grouping for RWAl were noted at both temperatures, they were generally more pronounced at $20^{\circ} \mathrm{C}$. In contrast, benefits of grouped development were largely restricted to the $24^{\circ} \mathrm{C}$ treatment for RWA2. For example, grouped RWA2 developed faster than their solitary counterparts on all three cultivars at $24^{\circ} \mathrm{C}$, with no such effects evident at $20^{\circ} \mathrm{C}$. In contrast, RWA1 developed faster in groups than in solitude on two of the three cultivars at $20^{\circ} \mathrm{C}$, but there were no significant differences at $24^{\circ} \mathrm{C}$. Differences in survival as a function of the grouping treatment were not significant at either temperature for RWA1, but there was a consistent trend toward improved survival in groups compared with singles, especially at $20^{\circ} \mathrm{C}$. Although RWA2 survived better in groups than in solitude on two of the three cultivars at $24^{\circ} \mathrm{C}$, survival was lower in groups than in solitary aphids on all three cultivars at $20^{\circ} \mathrm{C}$. The reduced survival of RWA2 in groups at $20^{\circ} \mathrm{C}$ would be consistent with competition for nutrients occurring among immature aphids when they fed together at a temperature that reduced their ability to manipulate plant suitability. 
RWA1

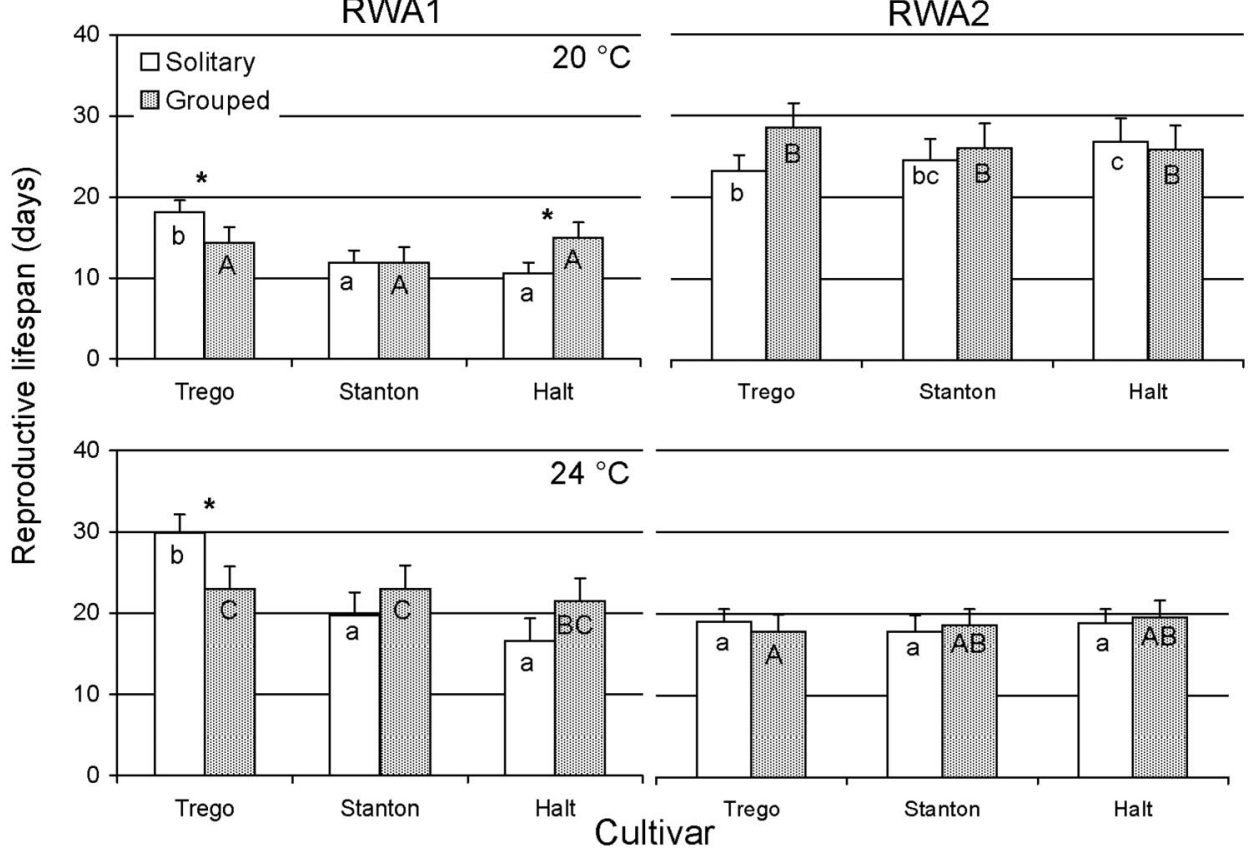

Fig. 4. Mean reproductive life spans (number of days from production of first to last nymph) (+SEM) of two D. noxia biotypes (RWA1 and RWA2) reared on three wheat cultivars at two constant temperatures. Solitary individuals developed in isolation on a wheat seedling and reproduced alone; grouped individuals developed in groups of 10 , and the first to mature was left on the plant to reproduce alone. Asterisks indicate significant differences (ANOVA, $\alpha<0.05$ ) between solitary and grouped aphids in a particular biotype-cultivar-temperature combination. Different lowercase letters indicate values for solitary aphids that were significantly different $(\alpha<0.05)$ within a temperature treatment; different uppercase letters indicate values for grouped aphids significantly different within a temperature treatment.

Only RWA1 showed effects of the grouping treatment on reproductive life span, which was shorter for groups than for singles on Trego at both temperatures. Given that the reproductive rate of RWAl groups was higher than for singles on this cultivar at both temperatures, the most likely explanation for the reduced longevity is a steeper decline in the condition of this susceptible cultivar toward the end of the experiment as a function of multiple aphids feeding. Notably, RWA1 reproductive rate was improved by the grouping treatment at both temperatures on all cultivars save for Halt at $20^{\circ} \mathrm{C}$. Although the reproductive rates of RWA2 were increased by grouped development exclusively at $24^{\circ} \mathrm{C}$, the magnitude of the increases seemed much larger than in RWA1. A similar pattern was evident for fecundity; the grouping treatment increased RWA2 fecundity on all three cultivars at $24^{\circ} \mathrm{C}$, but only on Trego at $20^{\circ} \mathrm{C}$, apparently as a function of longer reproductive life.

Because of the need to distinguish the exact fecundity of individual adults, the grouping treatment could only be accomplished during the prereproductive life of each aphid in the reproduction assay. Thus, the magnitude of fitness benefits measured may represent an underestimate of those occurring in naturally developing aphid colonies, assuming a greater degree of plant suitability might have been elicited had all aphids been left on the plant to reproduce together, and all daughters permitted to accumulate during the reproductive period of their mothers. Furthermore, the improved reproductive performance observed in aphids experiencing grouped development must have resulted from changes in plant suitability induced before their maturity that continued to benefit them throughout reproductive life. This would suggest that the wheat plants were unable to appreciably reverse the aphid-induced changes within the time frame of the experiment $(30-40 \mathrm{~d})$, even though infestation was reduced to a single adult aptera after $10-15 \mathrm{~d}$. The dosage-dependent nature of these induced changes is further illustrated by the significant positive regressions of group size at age of first reproduction on both fecundity and reproductive rate in RWAl: the bigger the group of surviving nymphs, the better the reproductive performance of the experimental aphid remaining on the plant. The lack of significance for such regressions in the case of RWA2 may reflect the fact that even small groups of this virulent biotype were able to elicit a maximal response from these young plants.

Initially, induction of suitability is probably localized within the plant, becoming systemic throughout the plant in later stages. Consequently, two D. noxia nymphs that developed together on the same leaf for at least $5 \mathrm{~d}$ were observed to develop faster than two nymphs that developed on different leaves of the same plant (Qureshi and Michaud 2005). Successful elicitation of plant suitability probably requires a signifi- 


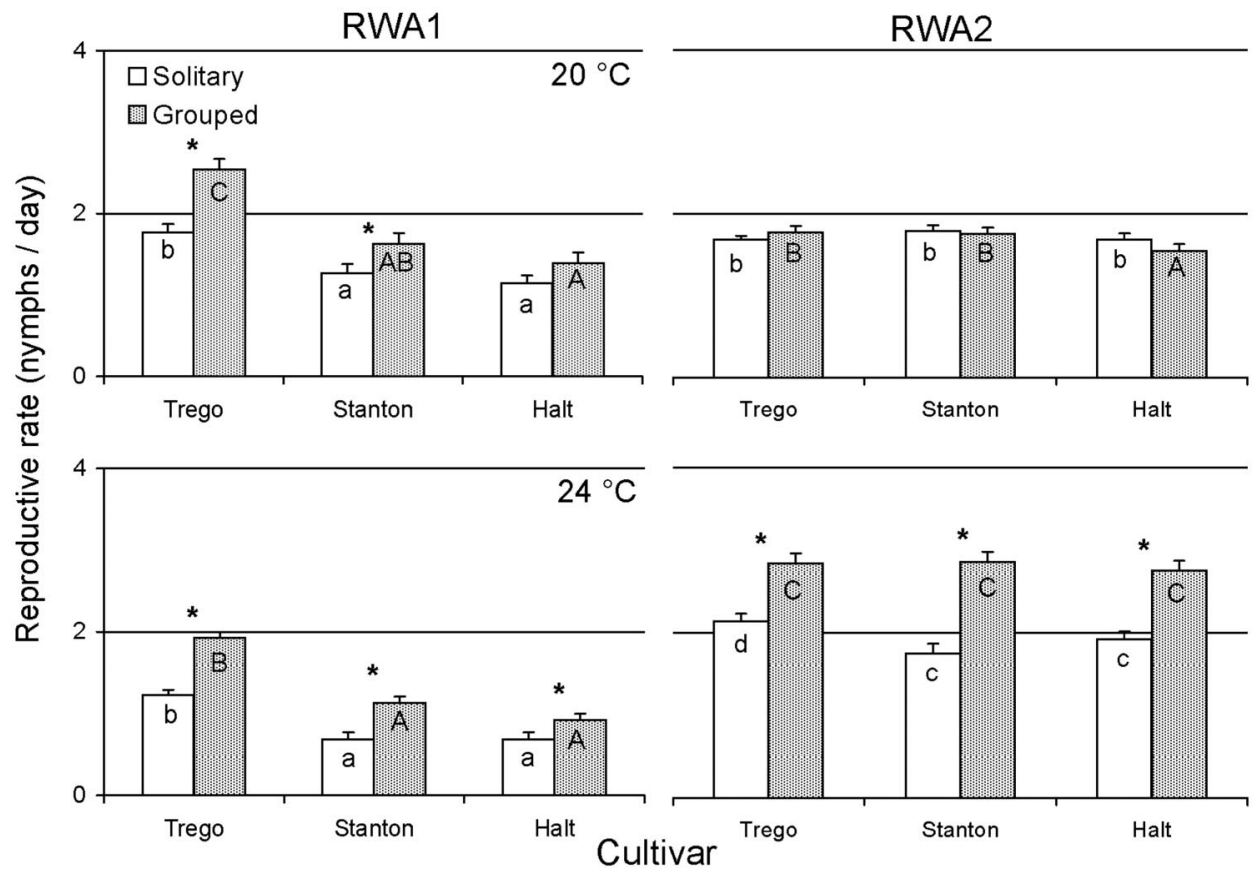

Fig. 5. Mean reproductive rates (number of nymphs per day of reproductive life) (+SEM) of two D. noxia biotypes (RWA1 and RWA2) reared on three wheat cultivars at two constant temperatures. Solitary individuals developed in isolation on a wheat seedling and reproduced alone; grouped individuals developed in groups of 10 , and the first to mature was left on the plant to reproduce alone. Asterisks indicate significant differences (ANOVA, $\alpha<0.05$ ) between solitary and grouped aphids in a particular biotype-cultivar-temperature combination. Different lowercase letters indicate values for solitary aphids that were significantly different $(\alpha<0.05)$ within a temperature treatment; different uppercase letters indicate values for grouped aphids significantly different within a temperature treatment.

cant investment in aphid saliva, initially injected at a localized site on a particular plant. Therefore, it is not surprising that aphids such as D. noxia move little after colonization and tend to form tightly aggregated col- onies to concentrate their efforts (Qureshi and Michaud 2005). Furthermore, because induction of plant suitability is a gradual, rather than immediate process, it is not easy for colonizing D. noxia, alate or

Table 2. ANOVA of fecundity, reproductive life, and reproductive rate for $D$. noxia that developed from first instars either in solitude or in groups of 10 (=grouping treatment) on each of three wheat cultivars (Trego, Stanton, and Halt) at each of two temperatures (20 and $24^{\circ} \mathrm{C}$ )

\begin{tabular}{|c|c|c|c|c|c|c|c|}
\hline \multirow{2}{*}{ Source of variation } & \multirow{2}{*}{$\mathrm{df}$} & \multicolumn{2}{|c|}{ Fecundity } & \multicolumn{2}{|c|}{ Reproductive life } & \multicolumn{2}{|c|}{ Reproductive rate } \\
\hline & & $F$ & $P$ & $F$ & $P$ & $F$ & $P$ \\
\hline Biotype & 1 & 213 & 0.0001 & 20.54 & 0.0014 & 470.18 & 0.0001 \\
\hline Replication & 9 & 0.61 & 0.7645 & 0.63 & 0.7474 & 1.20 & 0.3972 \\
\hline Replication (biotype) $=\operatorname{error}(\mathrm{a})$ & 9 & 0.94 & 0.4931 & 0.97 & 0.4678 & 0.56 & 0.8280 \\
\hline Temp & 1 & 3.21 & 0.0899 & 1.67 & 0.2128 & 3.31 & 0.0856 \\
\hline Biotype*temp & 1 & 0.24 & 0.6326 & 82.14 & 0.0001 & 198.58 & 0.0001 \\
\hline Rep*temp (biotype) $=$ error $(b)$ & 18 & 1.23 & 0.2360 & 0.73 & 0.7818 & 1.01 & 0.4493 \\
\hline Cultivar & 2 & 23.02 & 0.0001 & 2.81 & 0.0631 & 48.35 & 0.0001 \\
\hline Grouping treatment & 1 & 26.69 & 0.0001 & 0.07 & 0.7882 & 107.03 & 0.0001 \\
\hline Cultivar* grouping treatment & 2 & 0.19 & 0.8300 & 1.70 & 0.1856 & 4.23 & 0.0160 \\
\hline Biotype*cultivar & 2 & 20.59 & 0.0001 & 4.61 & 0.0112 & 27.94 & 0.0001 \\
\hline Biotype* grouping treatment & 1 & 0.00 & 0.9515 & 0.02 & 0.9000 & 0.51 & 0.4746 \\
\hline Temp*cultivar & 2 & 0.47 & 0.6274 & 0.66 & 0.5196 & 0.57 & 0.5680 \\
\hline Temp*grouping treatment & 1 & 5.42 & 0.0210 & 0.70 & 0.4046 & 26.79 & 0.0001 \\
\hline Biotype* cultivar*grouping treatment & 2 & 1.05 & 0.3512 & 4.96 & 0.0080 & 3.17 & 0.0445 \\
\hline Biotype*temp*cultivar & 2 & 0.26 & 0.7714 & 0.48 & 0.6188 & 0.93 & 0.3958 \\
\hline Biotype*temp*grouping treatment & 1 & 1.20 & 0.2754 & 1.10 & 0.2968 & 26.27 & 0.0001 \\
\hline Temp*cultivar* grouping treatment & 2 & 0.96 & 0.3855 & 0.89 & 0.4113 & 1.09 & 0.3391 \\
\hline Biotype*temp*cultivar*grouping treatment & 2 & 2.87 & 0.0593 & 0.63 & 0.5311 & 0.62 & 0.5371 \\
\hline Rep*trt (biotype*temp*cultivar) $=$ error $(\mathrm{c})$ & 180 & 1.01 & 0.4742 & 0.89 & 0.8214 & 1.06 & 0.3116 \\
\hline
\end{tabular}

Groups were reduced to single aphid when the first one matured and all virginoparae reproduced in isolation. Rep, replicate; trt, treatment. 
Table 3. Linear regressions of developmental time, fecundity, and reproductive rate on group size at first reproduction for two biotypes of D. noxia (RWA1 and RWA2) at each of two temperatures

\begin{tabular}{|c|c|c|c|c|c|c|c|c|c|c|}
\hline \multirow{2}{*}{ Variable } & \multicolumn{5}{|c|}{ RWA1 } & \multicolumn{5}{|c|}{ RWA2 } \\
\hline & $F$ & $P$ & b0 & $\mathrm{bl}$ & $r^{2}$ & $F$ & $P$ & b0 & $\mathrm{bl}$ & $r^{2}$ \\
\hline \multicolumn{11}{|l|}{$20^{\circ} \mathrm{C}$} \\
\hline Developmental time & 24.07 & $<0.001$ & 10.28 & -0.15 & 0.293 & 0.67 & 0.416 & 10.66 & -0.06 & 0.011 \\
\hline Fecundity & 16.03 & $<0.001$ & 4.27 & 3.21 & 0.217 & 0.24 & 0.629 & 38.78 & 0.78 & 0.004 \\
\hline Reproductive rate & 11.87 & 0.001 & 0.71 & 0.19 & 0.170 & 3.04 & 0.087 & 1.22 & 0.07 & 0.050 \\
\hline \multicolumn{11}{|l|}{$24^{\circ} \mathrm{C}$} \\
\hline Developmental time & 15.20 & $<0.001$ & 7.58 & -0.17 & 0.211 & & & & & \\
\hline Fecundity & 9.53 & 0.003 & 15.01 & 2.98 & 0.143 & 0.05 & 0.820 & 53.16 & -0.32 & 0.001 \\
\hline Reproductive rate & 17.00 & $<0.001$ & 0.78 & 0.12 & 0.230 & 0.06 & 0.802 & 2.73 & 0.13 & 0.001 \\
\hline
\end{tabular}

All replicates were initiated with 10 first instars and data were recorded for the first adult virginoparae to reproduce within a group, all others being removed on this date.

apterous, to effectively assess the potential suitability of a candidate host plant during a brief feeding bout. Thus, wheat cultivars with antibiosis are not initially avoided by colonizing D. noxia (Qureshi et al. 2005), likely because a single colonizing aphid must inject considerable saliva before being able to gauge the plant's response. It is notable also that benefits of the grouping treatment for RWAl were evident even on cultivars expressing antibiosis to this biotype, even though plant suitability was never raised to the level of the susceptible Trego, indicating that Dn4- and Dny-based resistance does not hinge on negation of the dosage-dependent effect of aphid feeding.

Messina (1993) conducted a series of experiments on D. noxia (RWA1) with an objective similar to ours-to demonstrate an effect of initial group size on per capita colony growth rate-and failed to detect any effect that would suggest "beneficial modification" of the wheat plant by the aphid aggregation. We can suggest a number of reasons, none mutually exclusive, that could explain this apparent lack of correspondence to our results. Messina (1993) used plants at the tillering stage (Zadoks GS 13-15) that were much more developmentally advanced than those in our experiments, and the lowest rate of aphid infestation used was two adult aphids, as opposed to a single first instar. Individual aphids were never followed for development or reproduction, and observations on whole colonies were made only after $7-10 \mathrm{~d}$. The experiments also finished with much larger numbers of aphids than did our experiments, so it is possible that negative effects of competition came into play before observations were made. This is further evidenced by the production of alates in these experiments; no alates developed in any of our experimental replicates.

Negative effects of crowding within high-density colonies have been construed to be an important stimulus for induction of wing development in various aphid species (Bonnemaison 1951, Sutherland 1969a, Michaud 2001), as have cues associated with host plant deterioration (Sutherland 1969b, Schaefers 1973, Müller et al. 2001). If plant suitability for D. noxia changes dynamically as a function of colony size, it likely follows a parabolic trajectory that increases to some asymptotic value, then decreases as plant productivity is overwhelmed. It is only during the latter period that wing development is cued in D. noxia (Baugh and Phillips 1991). It follows that $D$. noxia colonizing a susceptible plant will achieve larger colony size and higher fitness than those colonizing a resistant plant by virtue of converting more plant biomass into alate biomass within a shorter period, reducing the period of exposure to natural enemies. Any decrease in group size due to predation, parasitism or disease will delay this process directly by reducing aphid numbers and indirectly by reducing dosage-dependent effects on plant suitability that are likely to be especially critical during early stages of colony establishment. In addition, our results indicate that empirical estimates of fitness parameters derived from observations of solitary aphids should be interpreted cautiously, because they may overestimate developmental time and underestimate reproductive rate compared with values that aphids can achieve when they develop and reproduce in groups.

Although it was hoped that the grouping treatment would yield different results on resistant and susceptible plant cultivars and so provide insight into the mechanism of wheat resistance to $D$. noxia, such was not the case. The only significant two-way interaction term between cultivar and grouping treatment was for reproductive rate. The life history parameters of RWA1 seemed similarly impacted by resistance whether solitary or grouped aphids were considered within a temperature regime, and benefits of grouping were evident on both resistant cultivars. In contrast, temperature regime had a markedly asymmetric effect on group feeding benefits in the two biotypes. Thus, future research into group effects in aphids should measure their responses at more than one biologically relevant temperature. Resistant cultivars still may be usefully applied in such studies, because highly susceptible cultivars may be too easily induced by one or two aphids as young plants, possibly obscuring feeding benefits in small groups as seemed to be the case for the fecundity of RWA1 on Trego at $20^{\circ} \mathrm{C}$. Future work also could apply analytical chemistry techniques to identify specific nutritional compounds that are elevated in the phloem of multiply-induced plants.

In summary, the benefits of group feeding have been previously demonstrated in a number of aphid species, including A. pisum (Murdie 1969), A. fabae 
(Way and Banks 1967, Dixon and Wratten 1971), and B. brassicae (Way and Cammell 1970). However, the fitness benefits reported in these studies were relatively modest in comparison with those measured in the current study and were attributed to increased sink effects generated by aphid groups rather than to qualitative changes in nutritional content of the plant sap. The results from our reproduction assay rule out any possibility of a sink effect because aphids on singly- and multiply-induced plants both reproduced in solitude. We propose that the various beneficial effects of grouped development on aphid performance observed in these experiments are best interpreted as resulting from variations in the degree to which $D$. noxia were able to elicit increases in plant suitability in a dosage-dependent manner. We hypothesize that a maximum group size must exist, likely determined by plant size and condition, beyond which all positive effects of group feeding should gradually become replaced by negative effects as aphid competition for diminishing resources intensifies and alates develop.

\section{Acknowledgments}

We thank K. A. Shufran for providing us with a clone of RWA2. The manuscript benefited from reviews by E. Boyko, M. Mackauer, M. Brewer, and two anonymous reviewers. This paper is contribution no. 06-318J from the Kansas State Experiment Station.

\section{References Cited}

Ali, A. A., A. S. Rajab, and H. Al-Hussiani. 1985. 1985. Relative susceptibility of different wheat varieties to aphid infestation. J. Agric. Water Res. Res. 4: 25-39.

Basky,Z. 2003. Biotypic and pest status differences between Hungarian and South African populations of Russian wheat aphid, Diuraphis noxia (Kurdjumov) (Homoptera: Aphididae). Pest Manag. Sci. 59: 1152-1158.

Baugh, B. A., and S. A. Phillips, Jr. 1991. Influence of population density and plant water potential on Russian wheat aphid (Homoptera: Aphididae) alate production. Environ. Entomol. 20: 1344-1348.

Bonnemaison, L. 1951. Contribution to the study of factors inducing the apparition of winged and sexual forms in the Aphidinae. Ann. Epiphyt. 2: 1-380.

Cherqui, A., and W. F. Tjallingii. 2000. Salivary proteins of aphids, a pilot study on identification, separation and immunolocalisation. J. Insect Physiol. 46: 1177-1186.

[CASS] Colorado Agricultural Statistics Service. 2004. Colorado winter wheat cultivars - 2004 crop. (http: / / wheat. colostate.edu/links.html).

Dixon, A.F.G. 2000. Aphid ecology. Chapman \& Hall, London.

Dixon, A.F.G., and S. D. Wratten. 1971. Laboratory studies on aggregation, size, and fecundity in the black bean aphid, Aphis fabae Scop. Bull. Entomol. Res. 61: 97-111.

Dorschner, K. W., J. D. Ryan, R. C. Johnson, and R. D. Eikenbary. 1987. Modification of host nitrogen levels by the greenbug (Homoptera: Aphididae): its role in resistance of winter wheat to aphids. Environ. Entomol. 16: 1007-1011.

Haley, S. D., F. B. Peairs, C. B. Walker, J. B. Rudolph, and T. L. Randolph. 2004. Occurrence of a new Russian wheat aphid biotype in Colorado. Crop Sci. 44: 15891592.

Harvey, T. L., and K. D. Kofoid. 1993. Reproduction and survival of the Russian wheat aphid on sorghum. J. Kans. Entomol. Soc. 66: 81-85.

Jyoti, J. L., and J. P. Michaud. 2005. Comparative biology of a novel strain of Russian wheat aphid (Homoptera: Aphididae) on three wheat varieties. J. Econ. Entomol. 98: 1032-1039.

Jyoti, J. L., J. P. Michaud, J. A. Qureshi, and T. L. Martin. 2006. Virulence of two Russian wheat aphid biotypes to eight wheat cultivars at two temperatures. Crop Sci. 46: $774-780$.

Liu, X. M., C. M. Smith, B. S. Gill, and V. Tolmay. 2001. Microsatellite markers linked to six Russian wheat aphid resistance genes in wheat. Theor. Appl. Genet. 102: 504510.

Martin, T. J., A. Fritz, and J. P. Schroyer. 2001. Stanton hard red winter wheat. Kansas State University Agricultural Experiment Station Publ. L-921. (http://www.oznet. ksu.edu/library/crpsl2/1921.pdf).

Messina, F. J. 1993. Effect of initial colony size on the per capita growth rate and alate production of the Russian wheat aphid. J. Kans. Entomol. Soc. 66: 365-371.

Michaud, J. P. 2000. Development and reproduction of ladybeetles (Coleoptera: Coccinellidae) on the citrus aphids Aphis spiraecola Patch and Toxoptera citricida (Kirkaldy) (Homoptera: Aphdidae). Biol. Control 18: 287-297.

Michaud, J. P. 2001. Colony density and wing development in Toxoptera citricida (Homoptera: Aphididae). Environ. Entomol. 30: 1047-1051.

Miles, P. W. 1998. Aphid salivary functions: the physiology of deception, pp. 255-263. In J. M. Nieto Nafria, and A.F.G. Dixon [eds.], Aphids in natural and managed ecosystems. Proceedings of the Fifth International Symposium on Aphids, 15-19 September 1997, Leon, Spain. Universidad de Leon, Secretariado de Publicaciones, Leon, Spain.

Miles, P. W. 1999. Aphid saliva. Biol. Rev. Cambr. Phil. Soc. 74: 41-85.

Müller, C. B., I. S. Williams, and J. Hardie. 2001. The role of nutrition, crowding and interspecific interactions in the development of winged aphids. Ecol. Entomol. 26: $330-340$.

Murdie, G. 1969. Some causes of size variations in the pea aphid Acyrthosiphum pisum Harris (Hemiptera: Aphididae). Trans. R. Entomol. Soc. Lond. 121: 423-442.

Ni, X. Z., S. S. Quisenberry, J. Markwell, T. Heng-Moss, L. Higley, F. Baxendale, G. Sarath, and R. Klucas. 2001. In vitro enzymatic chlorophyll catabolism in wheat elicited by cereal aphid feeding. Entomol. Exp. Appl. 101: 159166.

Ni, X. Z., and S. S. Quisenberry. 2003. Possible roles of esterase, glutathione S-transferase, and superoxide dismutase activities in understanding aphid-cereal interactions. Entomol. Exp. Appl. 108: 187-195.

Powell, G. 2004. Sieve element salivation and the transmission to ingestion, pp. 479-483. In J. C. Simon, C. A. Dedryver, C. Rispe, and M. Hulle [eds.], Aphids in a New Millennium. Proceedings of the Sixth International Symposium on Aphids, September, 2001, Rennes, France. INRA Editions, Versailles, France.

Qureshi, J. A., and J. P. Michaud. 2005. Comparative biology of three cereal aphids on TAM 107 wheat. Environ. Entomol. 34: 27-36.

Qureshi, J. A., J. L. Jyoti, and J. P. Michaud. 2005. Differential colonization of wheat cultivars by two biotypes of 
Russian wheat aphid (Homoptera: Aphididae). Insect Sci. 12: 341-349.

Randolph, T. L., F. B. Peairs, M. K. Kroening, J. S. Armstrong,

R. W. Hammon, C. B. Walker, and J. S. Quick. 2003. Plant damage and yield response to the Russian wheat aphid (Homoptera: Aphididae) on susceptible and resistant winter wheats in Colorado. J. Econ. Entomol. 96: 352-360.

Sandström, J., A. Telang, and N. A. Moran. 2000. Nutritional enhancement of host plants by aphids - a comparison of three aphid species on grasses. J. Insect Physiol. 46: 33-40.

SAS Institute. 1999-2000. The SAS system for Windows, release 8.02. SAS Institute, Cary, NC.

Schaefers, G. A. 1973. The role of nutrition in alary polymorphism - an overview. Fruit World and Market Grower (January issue): 21-27.

Shufran, K. A., D. C. Margolies, and W. C. Black, IV. 1992. Variation between biotype E clones of Schizaphis graminum (Homoptera: Aphididae). Bull. Entomol. Res. 82: 407-416.

Smith, C. M., T. Belay, C. Stauffer, P. Stary, I. Kubeckova, and S. Starkey. 2004. Identification of Russian wheat aphid populations virulent to the Dn4 resistance gene. J. Econ. Entomol. 97: 1112-1117.

Stone, G. N., and K. Schönrogge. 2003. The adaptive significance of insect gall morphology. Trends Ecol. Evol. 18: $512-522$
Sutherland, O.R.W. 1969a. The role of crowding in the production of winged forms by two strains of the pea aphid, Acyrthosiphum pisum. J. Insect Physiol. 15: 1385-1410.

Sutherland, O.R.W. 1969b. The role of the host plant in the production of winged forms by two strains of the pea aphid, Acyrthosiphum pisum. J. Insect Physiol. 15: 2179 2201.

Telang, A., J. Sandström, E. Dyreson, and N. A. Moran. 1999. Feeding damage by Diuraphis noxia results in a nutritionally enhanced phloem diet. Entomol. Exp. Appl. 91: 403-412.

Tjallingii, W. F. 1995. Regulation of phloem sap feeding by aphids, pp. 190-209. In R. F. Chapman and G. de Boer [eds.], Regulatory mechanisms in insect feeding. Chapman \& Hall, New York.

Way, M. J. 1967. Intra-specific mechanisms with special references to aphid population. Symp. R. Entomol. Soc. Lond. 4: 18-36.

Way, M. J., and C. J. Banks. 1967. Intra-specific mechanisms in relation to the natural regulation of numbers of Aphis fabae Scop. Ann. Appl. Biol. 59: 189-205.

Way, M. J., and M. Cammell. 1970. Aggregation behaviour in relation to food utilization by aphids, pp. 229-247. In A Watson [ed.], Animal populations in relation to their food resources. Blackwell, Oxford, England.

Received 19 January 2006; accepted 12 May 2006. 